\title{
Hormone therapy for menopausal symptoms
}

In this editorial by Helen Roberts (BMJ 2012;344:e815, doi:10. 1136/bmj.e815), reference 1 (Shapiro et al) contains an error. The article cited was published in the Journal of Family Planning and Reproductive Health Care (and not "Family Plann" as published).
Cite this as: BMJ 2012;344:e1172

๑ BMJ Publishing Group Ltd 2012 